\title{
¿Villas y Hogares Seguros? Prevención del Delito en la Comuna de Estación Central, Chile
}

\author{
Roberto Lagos Flores \\ Norberto Salinas Astudillo \\ Chile
}

Fecha recepción: 20/12/20I2

Fecha aceptación: 22/03/20I2

\section{RESUMEN}

Este artículo evalúa una política de prevención del delito, basada en el sistema de alarmas residenciales en la comuna de Estación Central de Santiago de Chile, diseñada para su implementación en barrios con problemas de inseguridad y victimización. Se utiliza la metodología del estudio de caso evaluativo, con el levantamiento de información primaria de 250 hogares. Los resultados arrojan como evidencia que el sistema de alarmas logra resultados moderados en términos de victimización e importantes en percepción de temor; sin embargo, existen alcances diferenciados según los micro-sectores estudiados, por lo tanto, sus logros globales son heterogéneos.Además, la gestión comunitaria del sistema logra efectos complementarios: buen uso del sistema, satisfacción, conocimiento municipal, efectividad en el tratamiento de la situación-problema, entre otros puntos útiles para la evaluación de estas experiencias.

Palabras claves: delincuencia - prevención del delito - alarmas comunitarias municipalidades, Chile

¿Safe Villages and Homes? Crime Prevention in the municipality of Estación Central, Chile.

\section{ABSTRACT}

This paper evaluates a crime prevention policy, based on the residential alarm system in the municipality of Estación Central of Santiago, Chile, designed its implementation in neighborhoods with problems of insecurity and victimization. It uses the methodology of case study evaluation, collecting primary data from 250 households. The results shows evidence that the alarm system has achieved modest results in terms of victimization and importants outcomes in fear perception; however, there are different scopes depending on the studied micro - sectors 
and, therefore, its global outcomes are heterogeneous. In addition, the comunity management of the system achieves complementary effects: good use of the system, satisfaction, municipal knowledge, effectiveness in treating of the situation -problems,among other things useful for the evaluation of these experiences.

Key words: delinquency - crime prevention - community alarms - municipalities Chile

\section{Vilas e lares seguros? Prevenção do delito no município da Estação Central, Chile}

\section{RESUMO}

Este artigo avalia uma política de prevenção do delito, baseada no sistema de alarmes residenciais no município da Estação Central de Santiago do Chile, feita para sua implementação em bairros com problemas de falta de segurança evitimização. Utilizase a metodologia do estudo de caso avaliativo, com o levantamento de informação primária de 250 lares. Os resultados lançam como evidência que o sistema de alarmes lograefeitos moderados em termos de vitimização e efeitosimportantesem percepção de temor; entretanto, existem resultados diferenciados segundo os micro-setores estudados; portanto, seus alcances globais são heterogêneos. Além disso, a gestão comunitária do sistema gera efeitos complementários: bom uso do sistema, satisfação, conhecimento municipal, efetividade no tratamento da situaçãoproblema, entre outros pontos úteis para a avaliação destas experiências.

Palavras-chaves: Delinquência - prevenção do delito - alarmes comunitários - municípios, Chile.

\section{INTRODUCCIÓN}

En Chile durante los últimos años los problemas de delincuencia y criminalidad han estado en el primer lugar de las preocupaciones públicas. Organizaciones y personas han reaccionado a su presencia cotidiana y todos los sectores políticos han contribuido al diseño y al re-diseño de políticas públicas que tienen como objetivo la prevención, control y sanción del crimen. Desde los círculos académicos también han surgido propuestas destinadas a resolver los problemas generados por las manifestaciones permanentes de la delincuencia y la criminalidad en la vida social.

Uno de los agentes menos visibles en esta materia pero tal vez el que ha hecho los mayores esfuerzos ha sido el municipio chileno. De hecho, la Encuesta Nacional de Opinión Pública Municipal (ENOPM, 20I0) indica que la municipalidad debiera abordar como tema principal la "prevención de la delincuencia" y, según las 
mismas personas, el municipio es la institución que tiene la mayor capacidad para resolver los problemas de la gente a nivel local.

Las municipalidades han ido de manera gradual incorporando a su quehacer la gestión de acciones relacionadas con la prevención del delito y de colaboración con acciones de control del crimen. Han llevado a cabo iniciativas desarrolladas por el liderazgo de sus autoridades y sustentadas en unidades especializadas que se han conformado recientemente, también lo han hecho motivados por las disposiciones generales del gobierno central. Esto deja entrever que los municipios han ido adquiriendo altas dosis de responsabilidad, lo que se ve reflejado en el interés y apoyo de la propia comunidad (vecinos y organizaciones sociales) por resolver las dificultades que los aquejan relacionados con los temas de seguridad. Los agentes municipales que conviven a diario con el problema, conocen sus manifestaciones y se nutren del saber local relacionado con el fenómeno, han logrado cierta experticia y cuentan con algunas facultades para colaborar y contribuir con acciones concretas frente al tema, articulando posibles soluciones desde la perspectiva local. No obstante, las políticas vigentes surgidas con el Plan Chile Seguro 20 I0-20I4, ponen en entredicho el apoyo a los municipios en la gestión de la seguridad local, toda vez que, por ejemplo, se desperfila su rol en los fondos nacionales de seguridad, pues son un actor más en la competencia por estos recursos.

Dentro de este marco se desarrollan actualmente una serie de iniciativas municipales en gestión directa con el gobierno central y que involucra a vecinos organizados, algunas entrelazadas y articuladas de modo eficiente y otras más particulares y con menos impacto. De este modo, seguimos a variados especialistas que han puesto su mirada en la importancia de los gobiernos locales para enfrentar el problema actual de la criminalidad (Crawford, 1997; Vanderschueren, 2007;Dammert, 2007; Manzano, 2009), todos ellos presentan un diagnóstico común y ciertas premisas básicas para observar y examinar las acciones locales en la materia.

En este trabajo evaluamos, como parte de la estrategia nacional de abordaje de esta problemática, el sistema de alarmas comunitarias en tres villas de la comuna de Estación Central (villa España, villa Fernando Gualda y villa Gustavo Guevara) como caso de estudio emblemático de la seguridad pública urbana desde una perspectiva local. Con este propósito, en primer lugar, describimos el marco referencial de seguridad pública en el espacio local y las estrategias actuales para trabajar en prevención del delito; en segundo lugar, describimos el sistema de alarmas comunitarias como estrategia de prevención ampliamente utilizado en Chile, luego realizamos un análisis de datos proveniente de la información obtenida de hogares que ejecutan este tipo de programa local, en dos momentos de medición, uno al inicio del sistema(2009-2010), y el segundo a más de un año de instalado el sistema(20l0-20l I), lo que nos permite hacer comparaciones y evaluar la eficacia de esta estrategia.Finalmente, discutimos sus resultados y las conclusiones del estudio con el fin de que se constituya en una herramienta útil de aprendizaje para acciones futuras de la misma política local o de otras que se generan de modo descentralizo en otros puntos del país. 


\section{MARCO REFERENCIAL}

El Plan de Seguridad Pública "Chile Seguro" 2010-2014, posee ámbitos de continuidad y otros de cambio con la anterior Estrategia Nacional de Seguridad Pública 2006-2010 y reconoce como prioritarias todas las medidas encaminadas a lograr el anhelo de la seguridad pública y la calidad de vida de las personas. El problema de la delincuencia se instaura como un problema que afecta la calidad de vida de cada ciudadano en todo ámbito: hijos que no pueden tener un momento de esparcimiento en lugares públicos, estudiantes que se sienten inseguros aún dentro de sus colegios, barrios antes acogedores ahora amagados por la violencia, etc.

Este plan gubernamental demuestra que en cada delito cometido no solamente interviene una persona con intenciones de delinquir, sino que existen otros factores que los facilitan, como lugares públicos propicios, falta de medidas de seguridad, entorno físico desmejorado, entre otros. Así, cada uno de estos factores debe ser abordado con programas e iniciativas diseñadas para alterar, corregir o finalmente eliminar estas circunstancias, las que resultan transversales a cada momento y a cada delito que se comete.

La disminución de los delitos se deberá obtener desplegando una serie de acciones que se han agrupado en cinco áreas de intervención o ejes temáticos que son la base de los diferentes programas actuales. Estas cinco áreas, a saber: prevenir, proteger, sancionar, apoyar y rehabilitar además de los ejes transversales relacionados con la gestión de la información y la ejecución en el territorio, son, según el Plan de Seguridad 20 I0, imprescindibles para lograr los niveles de seguridad esperados, siempre enfocados a los requerimientos propios de cada territorio (Ministerio del Interior, 20I0).

Esta proposición se concreta,por ejemplo, en el caso de la comuna de Estación Central que tiene una problemática delincuencial caracterizada por su individualidad geográfica, de espacios públicos, de los orígenes del territorio comunal, su condición económica, social y cultural. Por ello es que susrequerimientos securitarios son finalmente particulares y por lo tanto los programas de intervención se deben orientar hacia territorios específicos aún dentro del espacio físico al interior de la comuna. Como dicen Muñoz y Godoy (2010) esta comuna es una de las puertas de entrada de Santiago y modelo de transformaciones territoriales. De ahí que para la presente investigación Estación Central, en el centro de la ciudad de Santiago, posee un carácter estratégico.

Estudiar un caso municipal mediante barrios afectados por problemas delictuales implica realizar un examen intenso y completo de una problemática o materia particular en un marco geográfico determinado y en cierto tiempo. Como indican Rodríguez y García (1999) este tipo de análisis consiste en un examen detallado, profundo y sistemático sobre un caso particular. $Y$ lo es todavía más cuando lainformación surge de las propias personas intervinientes en un proceso paulatino de prevención del delito y la inseguridad, como los que ocurren en estos tres barrios que estudiamos. 
La administración municipal de Estación Central, en concordancia con las prioridades gubernamentales, se propuso disminuir el porcentaje de hogares víctimas de delitos y reducir los delitos que se cometen en espacios públicos, para ello se apoyó en los Proyectos del Fondo de Apoyo a la Gestión Municipal (FAGM 2009-20 I0), orientados a la intervención de territorios comunales en una primera etapa con la instalación de cámaras de televigilancia y en una segunda etapa con la colocación de alarmas comunitarias en sectores con hogares altamente victimizados. Este trabajo fue realizado durante los años 2009 y 2010.

Los delitos que afectan a los hogares de la comuna han tenido variaciones a través de los años, disminuyendo en 2006, pero creciendo de forma gradual llegando a un aumento de I,3\% en la variación 2008-2009. En 2009 la victimización en hogares llegó a 36,7\%, en tanto en 2010 llegó a 38\%, porcentaje mayor al nivel país y de la región para mismo período de tiempo. El alto nivel de victimización en hogares genera naturalmente preocupación pública toda vez que este tipo dedelito es el que daña más gravemente al grupo familiar, ya que las personas reconocen el hogar como el sitio donde se sienten más protegidos de la delincuencia. Según el índice de vulnerabilidad socio delictual la comuna ocupa el lugar 13 con una puntuación de 82,6 (en escala de 0 a 100) el grupo más alto del ranking a nivel país (Araya, 2009).

\section{Alarmas comunitarias como estrategia preventiva}

Las autoridades comunales, encargados técnicos y la propia comunidad decidieron en 2009 aunar esfuerzos para la materialización de proyectos específicos. Uno de ellos destinado a combatir el delito que afectaba a los hogares, buscando prevenir el delito de robo a lugar habitado o destinado a casa habitación, mediante estrategias de alarmas comunitarias. Las estadísticas delictuales de la comuna identificaron ciertos sectores donde concretar esta estrategia con el fin de mantener y en lo posible disminuir estos delitos a hogares. El proceso involucró aprender de experiencias similares en las comunas de Cerrillos, Maipú, El Bosque y Santiago donde se habían instalado con anterioridad, como también de experiencias internacionales.

Luego de decidir la instalación de alarmas comunitarias en algunos sectores comunales, se diseñó una estrategia para sensibilizar a las personas, por ejemplo, informando sobre el real alcance del sistema preventivo, la importancia de la participación ciudadana para su desarrollo y las circunstancias sociales que permiten una mejor coordinación y gestión del sistema. Cabe destacar que según la experiencia municipal el uso dado al sistema de alarmas domiciliarias depende en gran medida de la dinámica social que se desarrolla a su alrededor. La cooperación entre las personas era la única forma en que vecinos de sectores con carencias económicas y sociales, podían obtener un elemento técnico para proteger su hogar, y que sintieran además que tienen las mismas posibilidades que otros vecinos de sectores acomodados y que son reconocidos por el gobierno y la municipalidad. 
Los sectores intervenidos en el período 2009-2010, y que son estudiados en esta investigación, fueron los siguientes:

\section{a) Primer Sector con alarmas: VILLA ESPAÑA}

Este primer sector está compuesto por las unidades vecinales $N^{\circ} 26$ villa España y $N^{\circ} 27$ correspondiente a un sector reducido físicamente de la Población Robert Kennedy, delimitado al poniente con Avenida Aeropuerto; al oriente con Avenida Las Rejas; Pasaje Quinchale y calle Palena, al norte Avenida 5 de Abril y al sur calle Lanceros del Rey y calle Río Quetro.

\section{a. I) Realidad delictual}

De acuerdo a la información disponible el sector presentaba una alta concentración de robos focalizado en el extremo poniente de villa España, en Av. Aeropuerto y en el extremo oriente con Av. las Rejas. De acuerdo a los demás delitos de mayor connotación social (DMCS), adicionamos la Calle Lanceros del Rey, Palena,Av. 5 de abril,Av. Las Rejas y la plaza ubicada en calle Río Quetro. Se estimó que la buena conectividad del sector ayudaba a la existencia de población flotante en el interior de las villas, utilizada como paso para acceder a las vías principales del sector y volver a sus hogares, lo que determina la acción delictual.Teniendo como base una Encuesta de Victimización e Inseguridad de 2009 (la que utilizamos como base del presente estudio evaluativo) previa a la instalación de las alarmas y realizada por el equipo municipal del proyecto, se constató una victimización de un $38,3 \%$ de los hogares por algún tipo de delito y que un $45 \%$ habían sido víctimas de un delito en sus hogares o supo de algún otro hogar en su entorno cercano. Asimismo el $43,8 \%$ de los entrevistados se sentía inseguro en su casa, un $60,4 \%$ inseguro en su calle o pasaje y un $62,4 \%$ inseguro en su barrio.

\section{a.2) Vulnerabilidad local}

De acuerdo a indicadores municipales el sector es catalogado en un "nivel medio" de vulnerabilidad socio-espacial, posee tres plazas y sus calles, veredas, pasajes e infraestructura se encuentran en buen estado al igual que las viviendas. La vulnerabilidad principal se produce por la población flotante y los vehículos que transitan, debido a que es paso obligado a sectores aledaños a locomoción colectiva para el desplazamiento a otras comunas de Santiago, además de la falta de poda de los árboles en algunos trayectos peatonales y en las tres plazas, que impide el paso de luz artificial en los espacios públicos. El sector es propiamente residencial, con pequeños comercios y servicios en las vías principales como Avenida Aeropuerto, Avenida Las Rejas Sur y Avenida 5 de Abril, sirviendo además de corredor de las poblaciones del sector norte y sur que convergen para acceder a la locomoción colectiva. 


\section{a.3) Uso del espacio y gestión local}

El centro cívico de este sector está emplazado en la junta de vecinos que se ubica en un espacio aproximado de 1.500 metros cuadrados, ubicado en Lanceros del Rey con Calle el Escorial, donde funciona la sede vecinal y una multicancha donde confluyen todos los grupos organizados a realizar sus actividades.

La información municipal no registraba un uso permanente por parte de grupos violentos y/o actividades delictuales.Los servicios municipales funcionaban eficientemente en el sector. El flujo peatonal y vehicular de esta zona es utilizada por las villas aledañas para acceder a las principales vías comunales. Según los datos municipales, en la Plaza Río Quetro, en el sector de la villa Robert Kennedy, se produce un gran flujo de personas, grupos de jóvenes y adultos, principalmente en las noches, que consumen alcohol y drogas, lo que hace vulnerables a los transeúntes y a las casas colindantes a ésta.

\section{b) Segundo Sector con alarmas: VILLA FERNANDO GUALDA Y VILLA GUSTAVO GUEVARA}

Este segundo sector está compuesto por las unidades vecinales $\mathrm{N}^{\circ} \mathrm{I5}$ I correspondiendo a la villa Fernando Gualda y 15-3 correspondiente a la villa Gustavo Guevara Vergara, delimitando al poniente con Avenida Las Rejas Sur; al oriente calle Las Araucarias y Las Magnolias; al norte con calle Titán y al sur con Avenida 5 de Abril.

\section{b. I) Realidad delictual}

De acuerdo a la información disponible del Ministerio del Interior los delitos a la propiedad se focalizaban en Pasaje Las Orquídeas y calle Las Magnolias, abarcando principalmente el perímetro delimitado por Avenida 5 de Abril y Avenida Las Rejas Sur, asimismo en calles interiores como Titán, Los Gladiolos y las dos plazas que posee esta zona.

Además se identificaron dos micro sectores de alta concentración delictual: los paraderos de locomoción colectiva por el sector norte ubicado en Pasaje Las Gardenias con Avenida 5 de abril y otro en el sector sur y de mayor flujo ubicado en Alameda con Avenida Las Rejas. Según la Encuesta de Victimización e Inseguridad que nos sirve de base de este estudio, en 2009 hubo un 38,3\% de hogares victimizados por algún tipo de delito y una victimización de un $45,5 \%$ de las personas en sus hogares o que supo de algún otro hogar en su entorno cercano, lo que demuestran un sector con una calidad desmejorada y alto nivel delictual.

Esta misma Encuesta indicó que el 63,7\% de las personas se sentía inseguro en su casa (un nivel muy alto comparativamente con su propia comuna), lo que aumentó a un $79,6 \%$ en su calle o pasaje y a un $71,2 \%$ en su barrio en general. 
El sector es predominantemente residencial, pero circundante tienen grandes vías como Avenida 5 de Abril,Avenida Las Rejas Sur y Avenida Bernardo O'Higgins con comercio mayor y mediano, centros hospitalarios, escuelas, con grandes afluencias de público, por ello su población flotante es alta y además es el lugar de escape de delincuentes cuando cometen algún delito en las calles principales.

\section{b.2) Vulnerabilidad local}

Existen dos plazas extensas en el sector, una ubicada en la villa Fernando Gualda que se encuentra en buen estado de conservación y buen mobiliario urbano, no obstante, presenta árboles frondosos que impiden el paso de la luz artificial al caer la tarde. La plaza ubicada en la villa Gustavo Guevara Vergara denominada Plaza El Cocodrilo, está en buen estado de conservación, con buen mobiliario, pero al igual que la anterior posee arbustos y árboles frondosos en ciertos sectores que impiden un adecuado control visual. La iluminación del sector es óptima y los servicios a la comunidad son eficientes, como asimismo el estado de las calzadas y aceras es aceptable en las calles principales, observándose un gran deterioro de éstas en los pasajes angostos. La zona posee una excelente conectividad a Avenida las Rejas y 5 de abril. Según los datos se puede caracterizar a este segundo sector con un nivel de "vulnerabilidad socio-espacial alto" debido principalmente a que es una zona de acceso a las principales avenidas, donde los delincuentes comenten ilícitos y escapan al interior de las villas.

\section{b.3) Uso del espacio y gestión local}

Los centros cívicos de este sector están emplazados en las juntas de vecinos de ambas villas, que se ubican en Titán con las Araucarias (villa Fernando Gualda), la cual posee una multicancha y la sede social ubicada en Los Gladiolos con las Magnolias (villa Gustavo Guevara). En estas confluyen todos los grupos organizados a realizar sus actividades como clubes de adulto mayor, grupos juveniles y culturales, centro de madres y deportistas.

Los otros centros de reunión son las dos plazas mencionadas anteriormente, utilizadas principalmente en el día por la comunidad, pero utilizada en la noche por grupos de jóvenes y población flotante para consumir drogas y alcohol. En el perímetro del sector principalmente en Av. Las Rejas con Av. 5 de abril existe comercio asociado a la población flotante y habitantes del sector, tales como supermercado, lugares de venta de comida al paso, bazares, farmacias, botillerías y carnicerías.

Finalmente, y después de descritos los sectores a intervenir, podemos indicar que para la ejecución del proyecto de alarmas comunitarias el Ministerio del Interior del Gobierno de Chile aportó \$28.150.000 y la Municipalidad de Estación Central $\$ 4.222 .500$, alcanzado la suma total de $\$ 32.372 .500$. 


\section{Características del sistema de alarmas}

Materialmente, la alarma comunitaria en estas villas corresponde a un elemento técnico que funciona con energía eléctrica y está compuesta por una fuente de poder, una sirena y una baliza estroboscópica, una batería de respaldo en caso de emergencia y su respectivo cargador, dispuestos en un gabinete metálico sellado para resistir a la intemperie. Externamente lo complementa un pulsador eléctrico que se coloca en el interior de la vivienda, el cableado que se integra a su circuito y un letrero de advertencia de "hogar protegido" con alarmas comunitarias. Como es fruto de una alianza gobierno y municipalidad este proyecto local no tienen costo para los beneficiarios.

Debido a las características de las viviendas se colocaron alarmas individuales y pareadas, es decir, dos casas con un solo gabinete con dos balizas que individualizan aquella que se encuentra en emergencia. Estas últimas son de un menor costo que las individuales como se puede ver en el Cuadro 2, donde además se enumeran los sectores donde se instalaron.

\section{Cuadro $N^{\circ} 2$ : Sistema de alarmas}

\begin{tabular}{|l|c|c|c|}
\hline \multicolumn{1}{|c|}{$\begin{array}{c}\text { SECTORES } \\
\text { INTERVENIDOS }\end{array}$} & Sistemas Individuales & Sistemas Pareados & Total \\
\hline Villa España & 482 & 100 & 492 \\
\hline Villa Fernando Gualda & 42 & 86 & 128 \\
\hline Villa Gustavo Guevara & 68 & 52 & 120 \\
\hline TOTAL & 592 & 238 & 740 \\
\hline
\end{tabular}

Fuente: Elaboración propia a base de proyecto municipal

Al comienzo de la ejecución del sistema los encargados municipales estimaron un rechazo a la participación entre un $20 \%$ y un $25 \%$ de los hogares, un porcentaje normal en una comunidad atomizada, ya sea por individualismo, falta de compromiso o temor. No obstante, al finalizar la instalación de los sistemas se pudo apreciar que la participación de los sectores beneficiados fue mayor a la esperada, en villa España hubo un $92 \%$ de adscripción al sistema, en villa Fernando Gualda un $98 \%$ y en villa Gustavo Guevara un $92 \%$ de participación.

\section{III.ANÁLISIS DE DATOS}

Cabe señalar que este estudio corresponde a los resultados de una investigación evaluativa de carácter cuantitativo mediante el uso de encuestas vecinales en los sectores descritos, del tipo evaluación ex-post. Siguiendo a Navarro, 
King, Ortegón y Pacheco la evaluación ex-post, en cualquiera de sus diseños "permitirá validar si efectivamente el programa está cumpliendo los objetivos para los cuales fue creado y podrá retroalimentar la ejecución del mismo para mejorar aquellos puntos sobre los cuales haya problemas" (2006: 10). A la cual, y como sustento interpretativo de resultados, se añadieron entrevistas en profundidad a actores sociales del territorio con el fin de buscar una mejor explicación de los hallazgos obtenidos.

Las preguntas que guían esta investigación dicen relación con: ¡Es efectiva la política de prevención local del delito en estos barrios urbanos, bajo la práctica del sistema de alarmas comunitarias para la consecución de los objetivos planteados en la intervención que le dio origen?; ¿cómo se ha modificado la percepción de temor al delito de los usuarios?; ¿han sido las acciones y actividades útiles o inadecuadas?; ¿cuál es la opinión de los usuarios respecto? y ¿en qué ha cambiado la situaciónproblema del temor y la delincuencia que dio origen a la estrategia local?

Para valorar el logro de los resultados generales de la prevención local del delito a través de la estrategia de alarmas comunitarias a nivel de hogares, en términos de eficiencia y efectos, recurrimos a un tipo de evaluación comparado entre la situación-problema inicial o de base (tiempo I) y la misma situación una vez ejecutada la acción (tiempo 2). De modo de intentar registrar fielmente los cambios acontecidos en la comunidad en el período de un año de ejecución de la estrategia local.

La situación de base se construyó como resultado de una Encuesta deVictimización e Inseguridad, de enero de 2009 ya referida anteriormente, en un diseño muestral de 740 casos-hogares, compuesta por población residente de los sectores intervenidos en la comuna. La situación de contraste con la que trabajamos es también una Encuesta deVictimización e Inseguridad, utilizada como instrumento de evaluación ex-post aplicada esta vez en las tres villas de los dos sectores de mayor población: I) villa España y 2) villa Fernando Gualda y villa Gustavo Guevara; intervenidos por el proyecto de alarmas comunitarias que sigue vigente en la actualidad. El diseño muestralen esta segunda medición fue de 250 casos-hogares, con un $5 \%$ de error y $95 \%$ de confianza.Ambos sectores generales corresponden al $80 \%$ de los hogares con sistemas de alarmas instalados fruto de esta estrategia en toda la comuna de Estación Central. Esto logra un nivel alto de representatividad de los sectores estudiados.

\section{Evaluación ex ante}

La situación de base se construyó con una muestra de hogares de los sectores villa Alessandri, villa Las Parcelas, Pasaje San Jorge,villa Fernando Gualda y Gustavo Guevara para el segundo semestre del año 2009. La muestra de 740 casoshogares estuvo constituida por un $65,3 \%$ de mujeres y en un $34,7 \%$ de hombres y formada mayoritariamente por población mayor de 60 años (42,9\%); luego por personas entre 4 I y 50 años ( $18,7 \%$ ); un 17,4\% de personas entre 52 y 60 años; un 
II,7\% de sujetos entre 31 y 40 años; un $6,7 \%$ por personas entre 21 y 30 años y menores de 20 años un $2,6 \%$ de los encuestados. Sin embargo, para los efectos de este estudio las comparaciones se realizan entre los dos sectores descritos: I) villa España y 2) villa Fernando Gualda y villa Gustavo Guevara.

En cuanto a la victimización en el momento previo al sistema de alarmas (medida siguiendo los criterios de las encuestas de victimización a nivel internacional) en la villa Fernando Gualda y Gustavo Guevara se presentó un mayor porcentaje de victimización, con un $\mathbf{6 0 , 7 \%}$ y Villa España con un $\mathbf{3 8 , 3} \%$. En lo referido a la ocurrencia de robo a la propiedad durante los últimos 12 meses, el primer sector de villa España tuvo un $45,5 \%$ de victimización por robo y la villa Fernando Gualda y Gustavo Guevara un 69,6\% (véase Gráfico I).

Gráfico I:Victimización por robo a la propiedad (tiempo I)

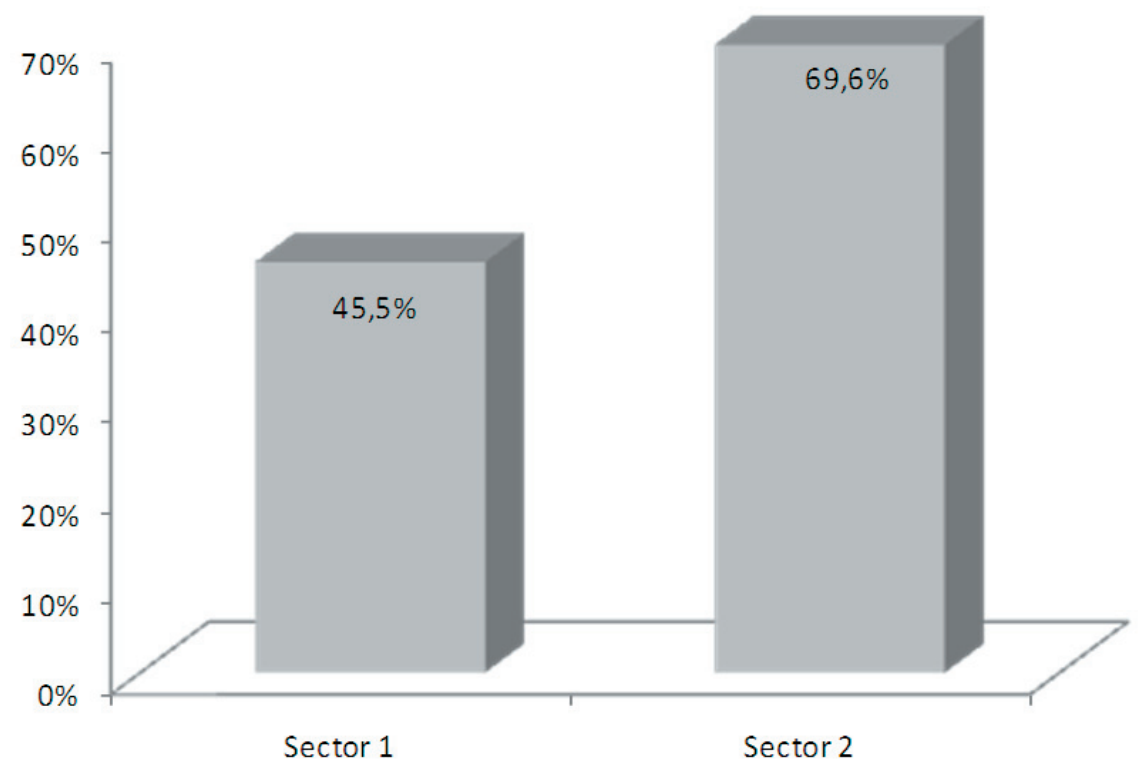

Fuente: Elaboración propia a base de Encuesta de Victimización 2009

En lo referido a la percepción de seguridad, los entrevistados que se sentían inseguros en su hogar (uniendo las categorías: "muy inseguro" e "inseguro" de la escala de medición) fueron los sectores de villa España con un 43,8\% y villa Fernando Gualda y Gustavo Guevara con un 63,7\%. 
Gráfico 2: Inseguridad en el hogar

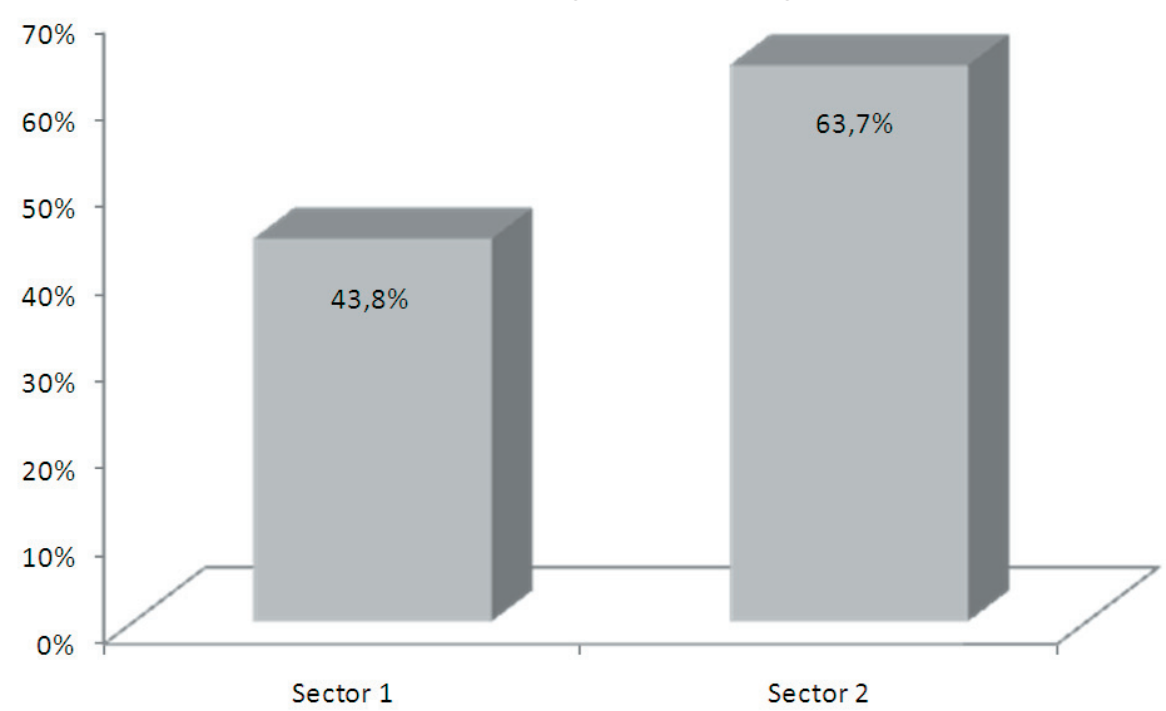

Fuente: Elaboración propia a base de Encuesta de Victimización 2009

Las personas se refirieron a la percepción de seguridad en su calle o pasaje, de esta forma, un $\mathbf{7 9 , 6 \%}$ de los encuestados de villa Fernando Gualda y Gustavo Guevara se sentían inseguros y muy inseguros y en villa España un $60,4 \%$ de los casos sentía la misma situación.

De naturaleza prospectiva se preguntó a las personas acerca de su disposición a participar en acciones comunitarias con el objetivo de mejorar sus niveles de seguridad, midiendo las opciones reales de intervención pública en la materia. En esta medición inicial los resultados fueron alentadores pues la comunidad manifestó abiertamente sus deseos de integrarse a estas tareas en su barrio. En las villas Fernando Gualda y Gustavo Guevara hubo un $94,6 \%$ de apoyo y en villa España un $73,8 \%$ de respuestas positivas.

\section{Evaluación ex post}

La evaluación realizada a un año de ejecución de la estrategia local (201020II) se construyó con una nueva muestra a nivel de hogares para los sectores seleccionados: villa España y villas Fernando Gualda y Gustavo Guevara, que como señalamos antes representan el $80 \%$ de la población que participó de la estrategia.

El universo es de 740 hogares. Se construyó un nuevo marco muestral, esta vez con 250 casos-hogares, con un 5\% de error y un $95 \%$ de confianza; grupo de estudio constituido por un $54,3 \%$ de mujeres y un $45,7 \%$ de hombres y formada mayoritariamente por población en un rango de edad que va desde los 26 a 98 años, muy similar a la muestra inicial (existe una media de 60 años, lo que permite inferir un grupo de sujetos con alto grado de experiencia y conocimiento local). El primer 
sector fue confeccionado con una muestra de 80 casos y un error de 7,3\% y el segundo sector con una muestra de 170 y un error de $7,3 \%$.

Ahora bien, el $31,7 \%$ de las personas encuestadas en sus hogares pertenecen a lavilla España y el restante 68,3\% a las villas Fernando Gualda y Gustavo Guevara. El $85 \%$ de los encuestados viven hace más de cinco años en el sector, lo que nos permite suponer un alto grado de conocimiento de su realidad social y asoman como fuentes válidas de información a nivel territorial. Lo que se suma al hecho que el $84 \%$ se declaran propietarios de sus viviendas y sólo un II\% de la muestra es "allegada" en los domicilios, lo cual reafirma su grado de conocimiento de la materia.

Además se reformuló el instrumento de recolección de información, manteniendo un grupo de preguntas originales que permiten la comparación entre la situación original de inseguridad pública y la situación posterior a la intervención. Este último instrumento mantuvo un apartado de caracterización territorial y social, sumado a un módulo de victimización, tipo de delito ocurrido, percepción de inseguridad, conocimiento de iniciativas municipales en el tema, evaluación de las mismas y finalmente un grupo de preguntas sobre el sistema de alarmas residenciales: referidas al uso dado, su evaluación, eficacia, tipos de problemas surgidos, cuidado y satisfacción.

Los más importantes hallazgos dicen relación con la disminución tanto de la victimización de hogares como de la percepción de inseguridad de las personas en la villa Fernando Gualda y en villa Gustavo Guevara (sector 2) y el mantenimiento de los niveles de la medición original en villa España (sector I).

\section{Gráfico 3: Comparativo de Victimización}

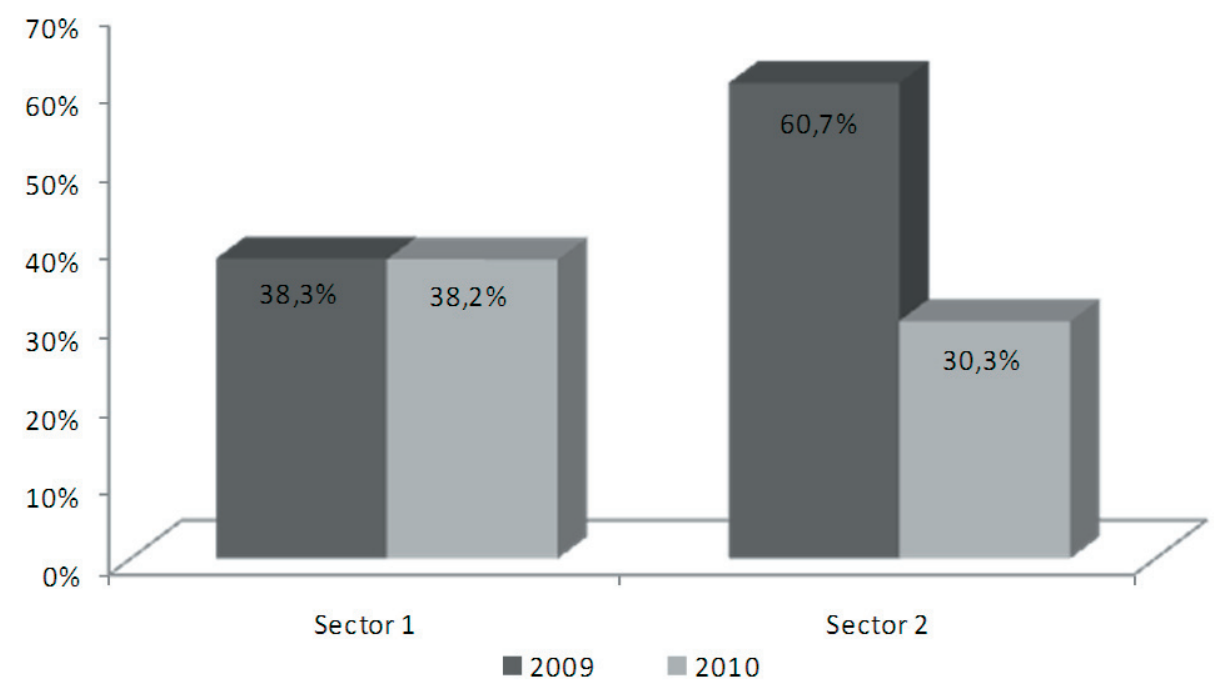

Fuente: Elaboración propia a base de Encuestas 2009-2010 
La victimización en hogares arroja resultados dispares en estas mediciones, comúnmente utilizadas para determinar los niveles de delincuencia en la población. Cabe destacar que para esta medición se utiliza la fórmula común de consultar en cada hogar visitado si durante los últimos doce meses:"¿Usted o algún miembro de su hogar fue víctima de algún delito?" El Gráfico 4 muestra los resultados por cada sector, donde el sector uno corresponde a villa España y el dos a villas Fernando Gualda y Gustavo Guevara.

Como se evidencia, la victimización bajó de un $60,7 \%$ a un $30 \%$ en las villas Fernando Gualda y Gustavo Guevara, siendo significativa su disminución y que en villa España prácticamente se mantuvieron los niveles iniciales (un 38,3\% en 2009 y un $38,2 \%$ en 2010). La victimización a nivel global bajó levemente teniendo en cuenta ambos sectores: de un $36,1 \%$ en 2009 a un $32 \%$ en 2010 , una cifra positiva, aunque dentro de los márgenes de error.

En tanto, luego del sistema de alarmas comunitarias la proporción de personas que indicó que la delincuencia aumentó en su barrio llegó a un $28 \%$ en villa España, un 54\% señaló que se mantuvo y un 17\% que disminuyó. En tanto, quienes indicaron que la situación de la delincuencia en su barrio aumentó llegó a un 19\% en las villas Fernando Gualda y Gustavo Guevara, aquellos que manifestaron que se mantuvo fue un $47 \%$ y quienes creen que disminuyó fue un $33 \%$. Como vemos las percepciones más positivas continúan estando presentes en el sector dos (villa Fernando Gualda y villa Gustavo Guevara).

Gráfico 4: Percepción de inseguridad

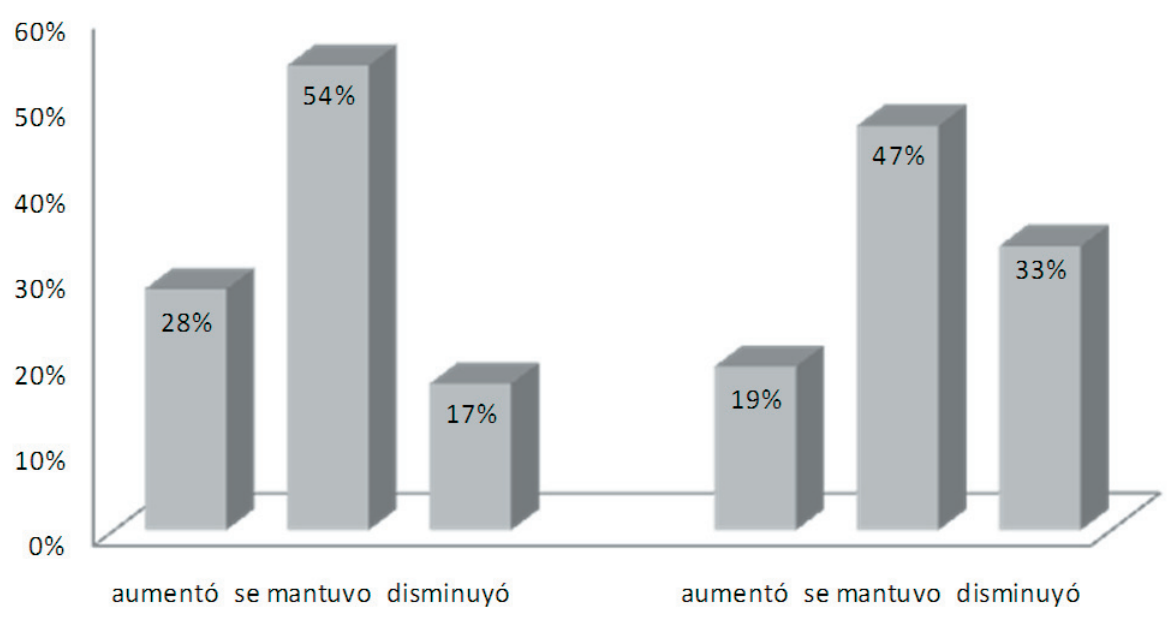

Sector 1

Sector 2

Fuente: Elaboración propia a base de Encuestas 2009-20I0 
Este punto es ilustrativo, pues tanto en la variable "victimización" como en "sentimiento de inseguridad", las personas de las villas Fernando Gualda y Gustavo Guevara presentan los datos más positivos, atribuibles al sistema de alarmas, menos delitos y mayor sensación de seguridad; en cambio, en villa España, si bien hay aspectos de mejoría, como un $17 \%$ de residentes que dicen que mejoró la seguridad a un año de instalada la política, las opiniones son muy similares a la situación de base 2009 y, por ende, es difícil presumir cambios producto del sistema.

A nivel de ambos sectores el $43 \%$ de los hogares fue víctima de un delito una sola vez, un $32 \%$ dos veces y un $24 \%$ más de dos veces; este hecho coincide con la evaluación de la victimización a nivel general en el país en cuanto a los altos niveles de revictimización en hogares.

Los delitos más frecuentes en estos sectores de Estación Central siguen siendo los de la primera medición, aunque en menor cantidad de sucesos, a saber, del grupo de $32 \%$ de hogares victimizados el $54 \%$ sufrió un robo desde su hogar, el resto (robo por sorpresa -“lanzazo"- con un 30\%, robo con violencia en un $7 \%$ y robo de automóvil y sus accesorios con un $2 \%$ y un $3 \%$ respectivamente) pueden ser considerados como delitos fuera de las residencias, al menos no posibles de atribuir al sistema de prevención evaluado.

De modo desagregado volvemos a ver que la situación de inseguridad sigue siendo mayor en villa España (sector I) pues el 68\% de los delitos ocurridos en el último tiempo corresponden a robo en hogares, en tanto, en villa Fernando Gualda y villa Gustavo Guevara de los delitos ocurridos, el $50 \%$ corresponde a mismo tipo de robo en casas.

En resumen, esta primera aproximación nos revela que la victimización bajó en el sector dos y se mantuvo en el sector uno; también, que la percepción de inseguridad bajó en villas Fernando Gualda y Gustavo Guevara y se mantuvo en villa España. Si bien los delitos bajaron en todos los sectores se mantienen como prioridad los robos a hogares en todo el territorio estudiado.

En un segundo plano, en esta nueva evaluación las personas calificaron con nota de I a 7 la seguridad de su barrio, como muchas encuestas evaluativas lo hacen para intentar medir apreciación personal y valoración respecto de la política. Las principales opciones muestran valores regulares y buenos sobre este ítem, por ejemplo, el $34,5 \%$ de las personas le puso nota 5 a su barrio, un $25,6 \%$ nota 4 y un $22,7 \%$ nota 6 . Son los grupos más altos y con calificaciones más positivas.

Según cada sector intervenido podemos indicar que en villas Fernando Gualda y Gustavo Guevara el 68,2\% de los habitantes calificó con notas sobre 5 a su barrio, por el contrario, en villa España la percepción es distinta, pues la mitad de los entrevistados la califica sobre 5 , pero la otra mitad bajo esa nota, dando a entender nuevamente resultados dispares entre ambos sectores intervenidos.Villa España como el sector más crítico e inconforme. 
Gráfico 5: Calificación al barrio (Nota en materia de seguridad)

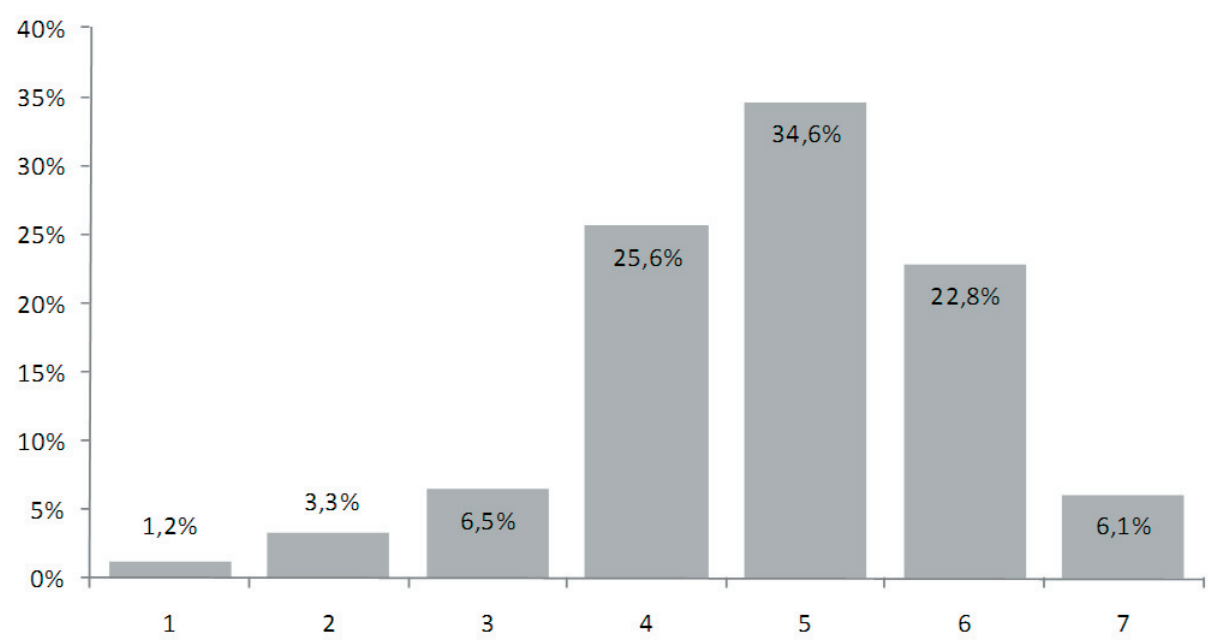

Fuente: autores a base de Encuesta de Victimización 2010

Para profundizar en la relación entre el municipio y los usuarios de este servicio de prevención podemos señalar que el $65 \%$ de los residentes, de los dos sectores estudiados, conocen las iniciativas en seguridad tomadas por su municipalidad, más allá del puntual sistema de alarmas que analizamos.

Este hecho revela que la evaluación de los municipios supera los niveles regulares de conocimiento, contrariamente a lo que pudiera suponerse. Para los dos sectores analizados, la distribución es la siguiente: en villa Fernando Gualda y villa Gustavo Guevara el 64\% de los vecinos las conocen y en villa España el 66,7\%. A todas luces un buen punto de partida para la gestión municipal en materia de prevención y control del delito.

Gráfico 6: Conocimiento de iniciativas municipales de seguridad

\section{Sector 1}

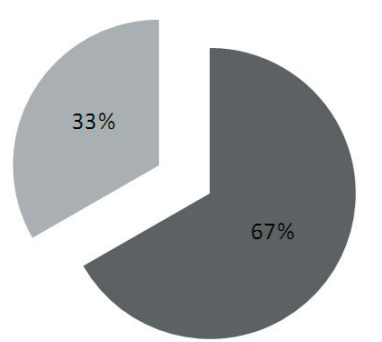

- Conoce

- No conoce

\section{Sector 2}

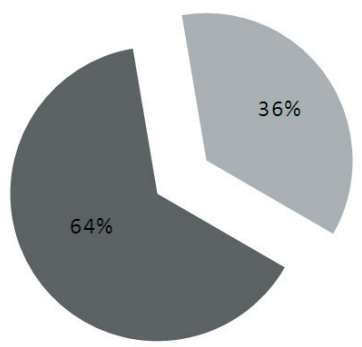

Fuente: autores a base de Encuesta de Victimización 2010 
Ahora bien, y como se observa en el siguiente Gráfico 7, las personas que conocían las iniciativas en seguridad tomadas por su municipalidad (el 65\% de la población) las calificaron en cuatro niveles de aprobación, desde muy malas (acciones inadecuadas) hasta muy buenas (acciones convenientes). A este respecto, el $72 \%$ de las personas las evaluó como buenas, un valor altísimo para cualquier evaluación de desempeño en el sector público. Visto de otro modo, un $90 \%$ de las personas que representan hogares con sistemas de alarmas instalados calificó como "buenas" o "muy buenas" las iniciativas de seguridad de su municipio.

De modo desagregado, los porcentajes de aprobación en cada sector identificado son muy similares, la calificación como "buena" de las iniciativas municipales en villa Fernando Gualda y villa Gustavo Guevara es de un 67\%, en tanto, en villa España es de un $79 \%$.

Este hecho estaría demostrando una vez más indicios de la valoración positiva que hacen los sujetos de las acciones del municipio en prevención y seguridad. Donde al parecer la victimización real y la percepción de inseguridad que se marcaba bastante diferenciada entre ambos sectores estudiados no tienen mayor distancia a la hora de evaluar al municipio. Este aparece como un agente local fortalecido en consonancia con su cercanía a las personas de estas villas.

Gráfico 7: Evaluación de iniciativas tomadas por su municipio en temas de seguridad

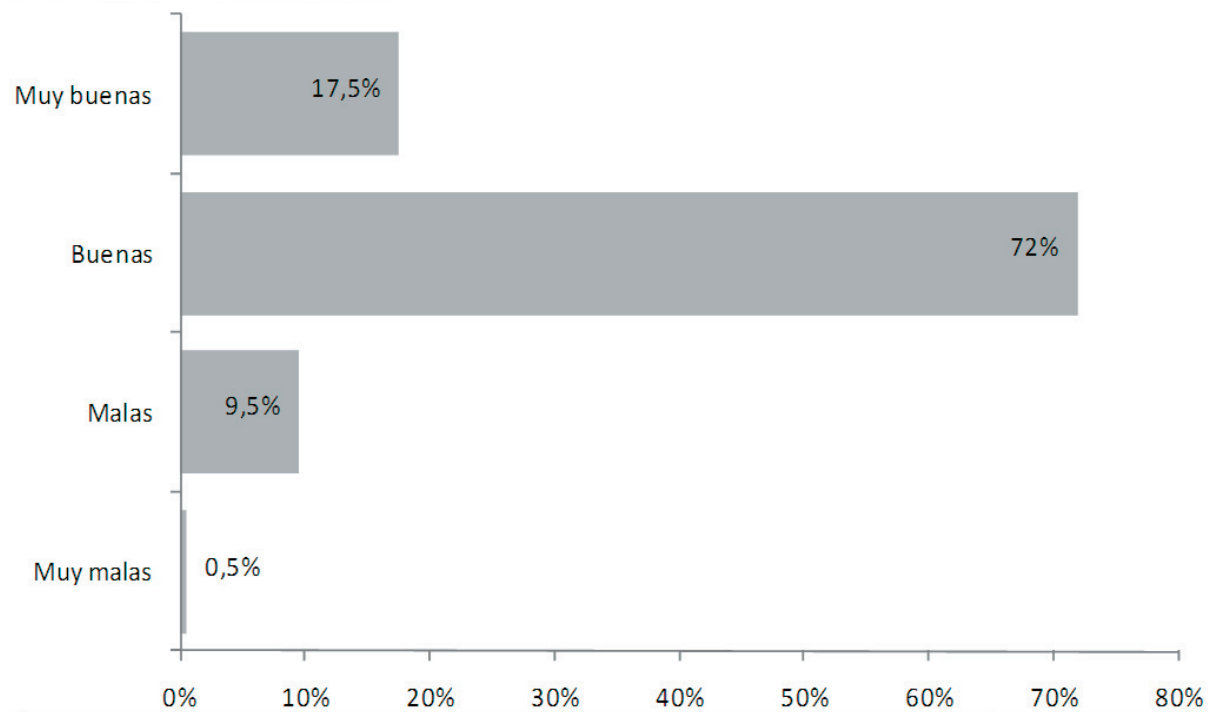

Fuente: autores a base de Encuesta de Victimización 2010 
Pasando específicamente al sistema de alarmas comunitarias, podemos demostrar que a un año de ejecutado el proyecto los índices de aprobación son muy altos (véase Gráfico 8). La mayoría de las personas, que representan hogares, indican que las alarmas en sus residencias fue una buena iniciativa, entendida como un proyecto de prevención.

Gráfico 8: Opinión del sistema de alarmas

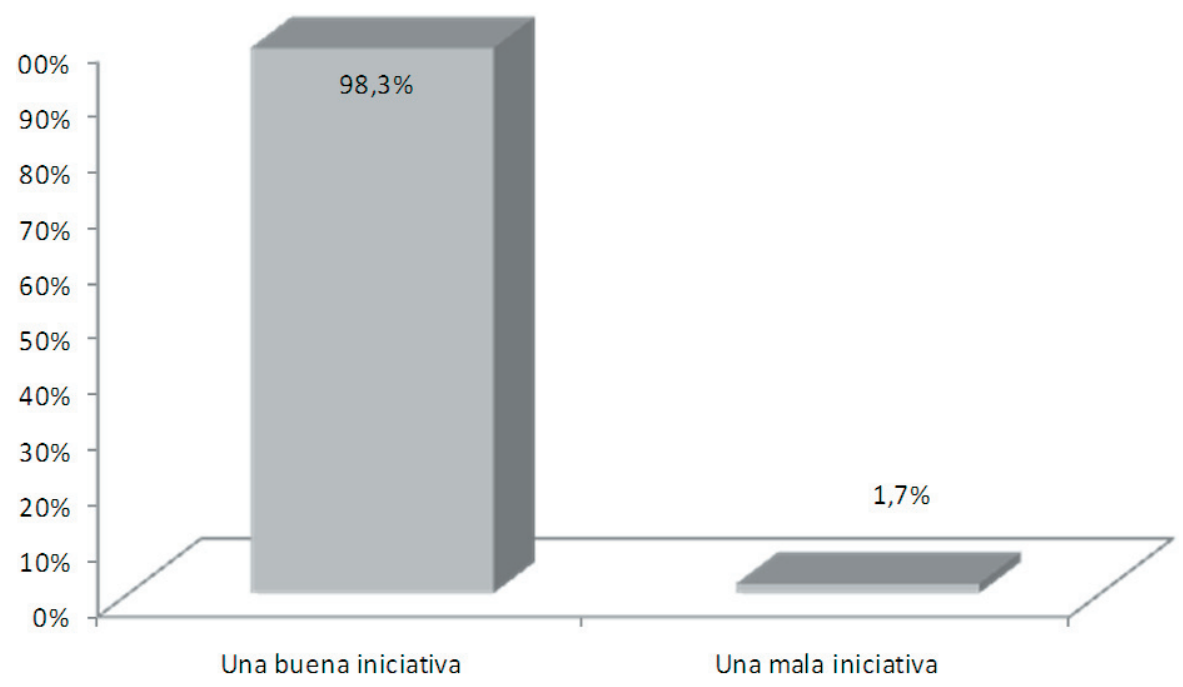

Fuente: autores a base de Encuesta de Victimización 2010

La alta aprobación, que a todas luces no es discriminante al menos numéricamente, muestra un sentir totalmente compartido en la población y vuelve a desechar las diferencias que muestran los dos sectores analizados en términos de victimización e inseguridad, es decir, más allá de los resultados directos del sistema, las alarmas son adecuadaspara el territorio. Del mismo modo, las personas encuestadas indicaron que se trata de una iniciativa que ayuda y contribuye a prevenir la delincuencia (93\% de sujetos), o sea, que según los propios sujetos este sistema trabaja directamente en la problemática que la originó. Como vemos un nuevo aspecto que reafirma la valoración positiva del sistema y su utilización directa en medidas de protección y prevención.

Sin embargo, en materia de uso del sistema los resultados difieren. En efecto, durante los últimos seis meses en general muy pocas familias han utilizado el sistema de alarmas ante un hecho de inseguridad o peligrosidad delictual que haya afectado sus residencias. Lo que no debilita la existencia del sistema preventivo, sino que muestra una característica clave del tema: es un dispositivo para disuadir que está presente como un objeto probable de ser usado, que otorga sensación de protección, pero que finalmente no se usa por cuanto los hechos delictuales en sí son escasos. 
Gráfico 9: ¿UD. o alguien de su familia ha usado el sistema de las alarmas?

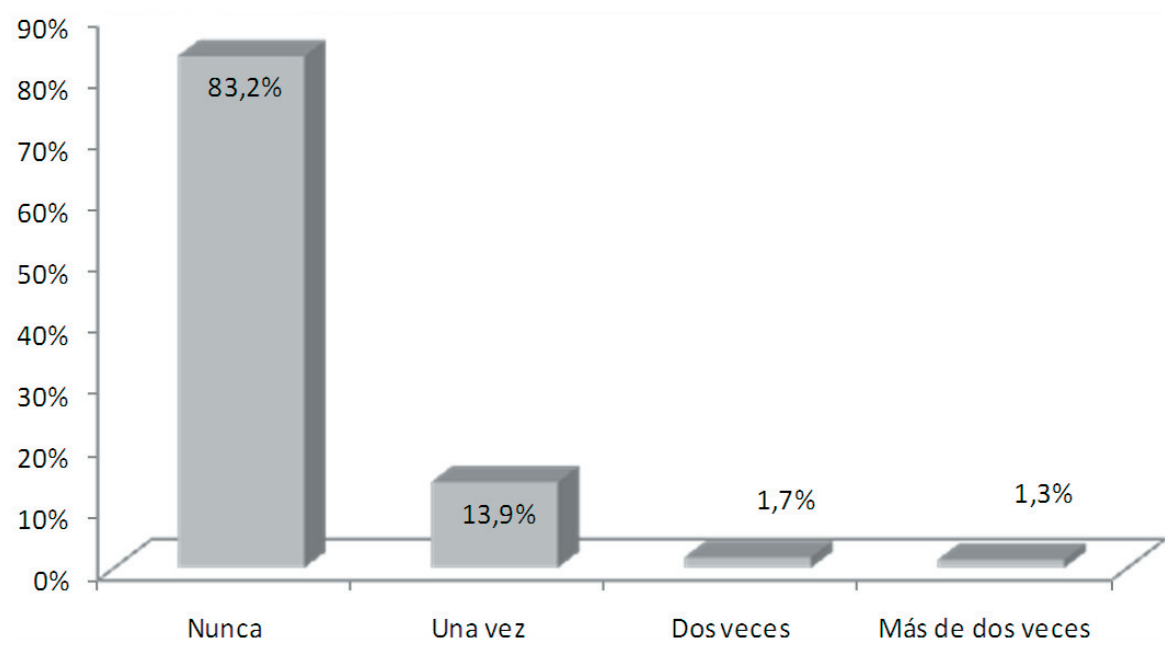

Fuente: autores a base de Encuesta de Victimización 2010

Como vemos en el gráfico anterior, el $83 \%$ de las personas no ha utilizado el sistema de alarmas domiciliarias, lo que hablaría de un servicio en desuso, poco útil. Sin embargo, la sola existencia del sistema y la gestión comunitaria que se desarrolla en su entorno es lo que valora la ciudadanía y cumple el objetivo que le dio origen. Además es llamativo que de todos modos más del I5\% de las personas las ha utilizado más de una vez, ello permite inferir que algunos sujetos ya poseen un cierto nivel de conocimiento práctico respecto del sistema, sus protocolos de uso y resultados concretos en el espacio local, se presume que ya han respondido a hechos delictivos. En villa España un $12 \%$ de los hogares las ha usado alguna vez y en las villas Gustavo Guevara y Fernando Gualda un $18,4 \%$.

Ahora bien, sin perjuicio de que los consultados hayan utilizado o no el sistema de alarmas comunitarias, a un año de su ejecución poseen opiniones respecto del problema que originó la intervención, o sea, respecto de la situación de base. Para ello, preguntamos a los beneficiarios si transcurrido este tiempo, el problema del temor al delito y de la victimización en sus hogares ha cambiado y en qué aspecto.

El Gráfico 9 muestra que el $57 \%$ de los hogares indica que se redujo el problema que dio origen a la intervención, una cifra positiva para cualquier política en tan corto plazo, además un $37 \%$ indicó que el problema se mantuvo, lo que podría expresar cierta disconformidad con los resultados de la intervención o más bien incertidumbre desde el punto de vista de la eficacia.

No obstante, hay un $4,8 \%$ de personas que indicó que se erradicó el problema lo que no deja de ser interesante para los ejecutores de la política local y para quienes evaluamos un proceso de estas características. Habría resultados directos sobre la situación original y éstos los avalarían un grupo numeroso de 
personas. Cabe destacar que las medidas de prevención situacional documentadas justamente coinciden en el establecimiento de estos logros debido al impacto social que generan, las personas sienten la seguridad y se la adjudican tanto al componente técnico (alarmas, cámaras o rejas) y al componente humano (organización vecinal), generando confianza, solidaridad y tranquilidad frente a los riesgos delictuales.

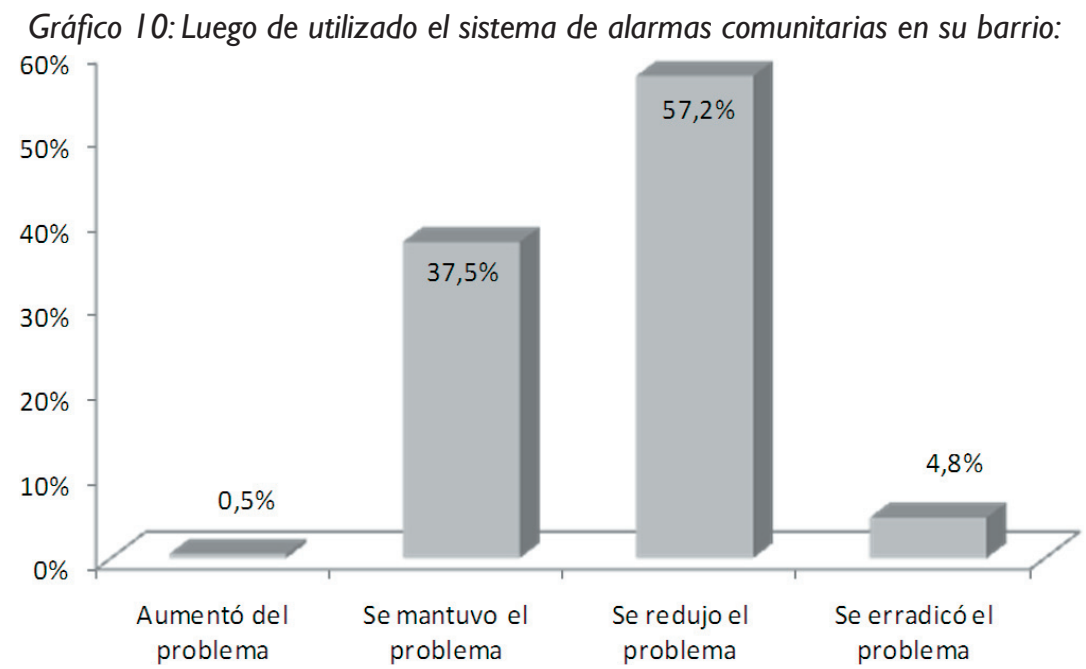

Fuente: autores a base de Encuesta de Victimización 2010

Siguiendo en la evaluación comunitaria de las alarmas, las personas indicaron los cambios observados por ellos una vez instalado el sistema, mediante la pregunta "¿Cómo cree UD. que ven el barrio las personas que viven fuera de él?" Las respuestas indican una evaluación positiva nuevamente en ambos sectores (Gráfico II).A nivel total, el $82 \%$ cree que son vistos por el resto de las personas como un lugar más seguro que antes, en cambio, un $17,7 \%$ cree que se percibe como más peligroso.

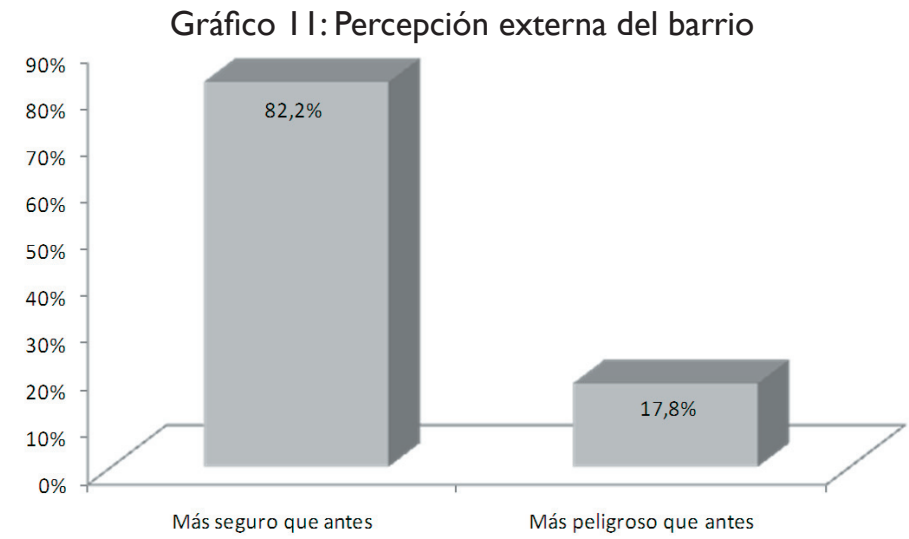

Fuente: autores a base de Encuesta de Victimización 2010 
De modo desagregado, podemos decir que no existen grandes diferencias por cada sector estudiado, tanto villa España como en villa Fernando Gualda y villa Gustavo Guevara las opiniones favorables superan el 80\%. En villa España el 82,4\% considera que el resto de las personas lo perciben más seguro que antes (un 17,6\% más peligroso que antes, lo que también puede ser leído con preocupación). En las villas Fernando Gualda y Gustavo Guevara el porcentaje también llega a $82 \%$.

En este estudio quisimos conocer, más allá de los logros de resultados y de satisfacción de los propios usuarios respecto del tema, cuáles son los principales problemas que existen en torno a la utilización y mantención de este tipo de sistema preventivo. Como es un modelo de gestión comunitaria de la seguridad, las respuestas tienen variadas opciones, sin embargo, algunas son bastante decidoras $y$ motivan a la reflexión y a la investigación posterior.

En efecto, según se puede ver en el Gráfico 12, si bien las personas manifestaron escasos problemas relacionados con el sistema de alarmas, aquellos que se inclinaron por alguna opción indicaron que lo principal estaba en la falta de coordinación entre los propios vecinos respecto del uso de las alarmas, a decir verdad, un $50 \%$ de las personas de estos barrios indican que el principal problema radica en la desorganización de los propios vecinos para operar el sistema. Según la mayoría es el tema más perentorio a resolver.

En segundo lugar, un $28,8 \%$ consideran que la dificultad está en la poca coordinación con Carabineros. En tercer lugar, un $8 \%$ señala problemas en la mantención de las alarmas. No obstante, las posiciones mayoritarias dicen relación con el modelo de gestión entre actores necesarios de responder frente a la operatoria del sistema, es decir, las dificultades del componente humano en la prevención aparecen como las de mayor importancia y es donde se debería intentar opciones de mejora. Claramente las personas piden más compromiso de sus propios vecinos y de la policía que los protege, es decir, propenden hacia una visión sistémica de cómo debe operar la prevención local.

Gráfico I2: Principal problema del sistema de alarmas

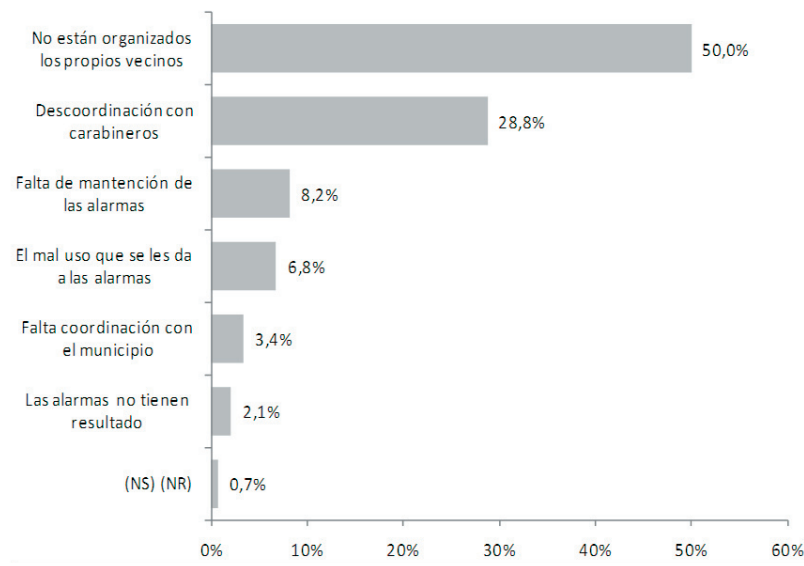

Fuente: autores a base de Encuesta de Victimización 2010 
Esto es corroborado con la opinión de informantes claves del propio sector, pues algunos agentes sociales indicaron que los tiempos de respuesta y de vigilancia policial no se condicen con los compromisos adquiridos previos al sistema de alarmas, igualmente la participación de la ciudadanía es oscilante y hay tiempo en que el individualismo predomina, pero luego, ante determinados hechos negativos como ilícitos o positivos como actividades comunitarias, vuelve la organización social.

En otra medida de los efectos logrados mediante el uso de este sistema está la percepción de los entrevistados respecto de si, como una consecuencia de la instalación de estos sistemas y como un objetivo a lograr de la intervención, la comunicación entre los propios residentes se mantuvo, decayó o mejoró. Según los estudios actuales este es un indicador de eficacia colectiva, premisa de base necesaria de lograr y que tiene impacto positivo en la prevención del delito.

A este respecto, un $63 \%$ indicó que "se mantuvo" la comunicación entre los vecinos beneficiados y un $31 \%$ que “mejoró”. Esta situación, sin duda, da a entender un efecto positivo del sistema de alarmas en un elemento que puede ser visto como adicional, pero imprescindible en toda política pública local.

\section{Gráfico 13: Comunicación vecinal}

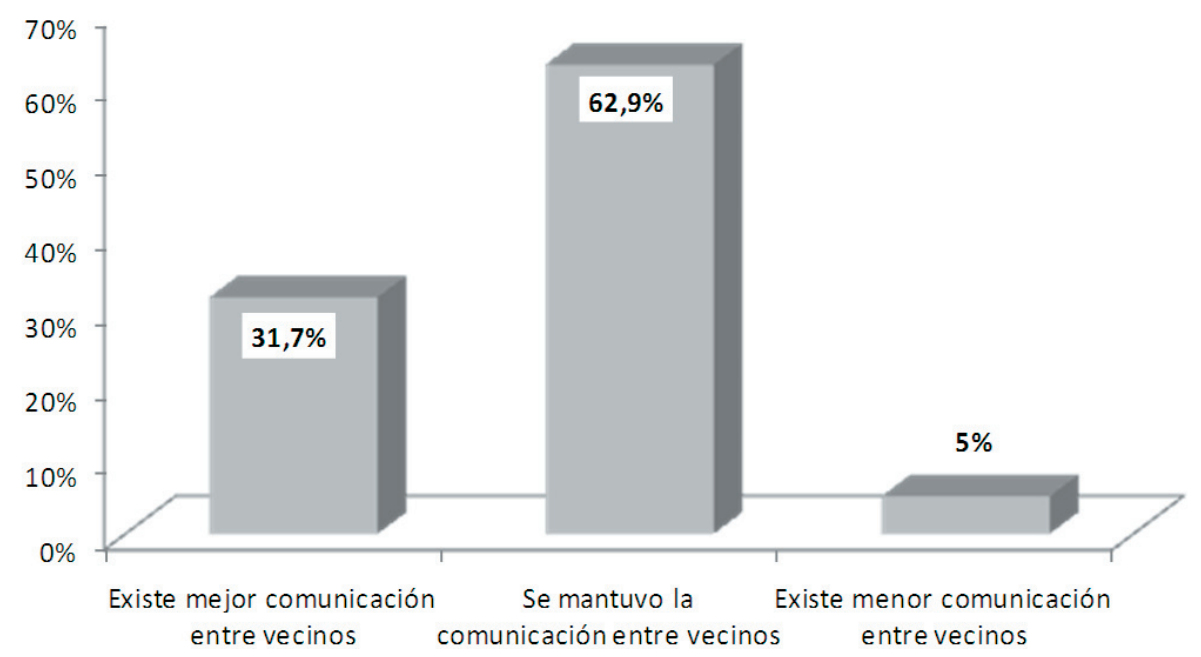

Fuente: autores a base de Encuesta de Victimización 2010

En una aproximación global al uso dado al sistema de alarmas comunitarias en el vecindario, las personas indicaron que en general ha habido corrección y adecuación de los vecinos a las normas y protocolos de usabilidad del sistema en su conjunto. No existen grandes visiones críticas.

De este modo, un 59\% indicó que ha habido un "adecuado" uso del sistema y un $38 \%$ un "muy adecuado" uso. 
Gráfico I4: Grado de conformidad con el uso del sistema

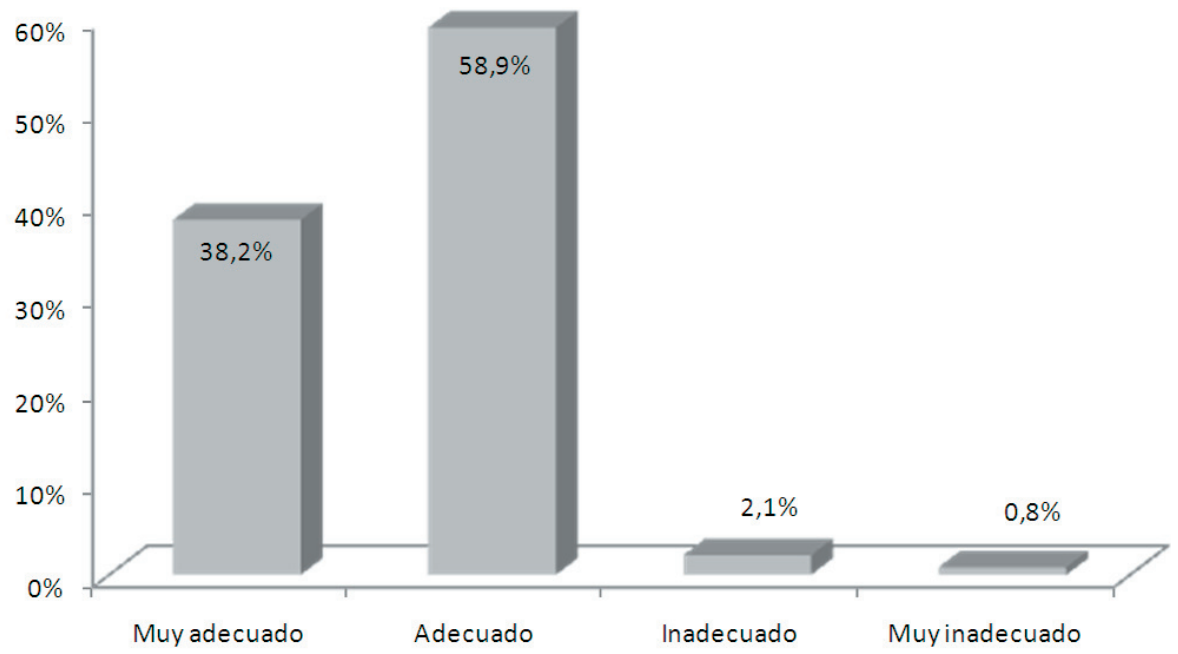

Fuente: autores a base de Encuesta de Victimización 2010

Como medida final de la evaluación de la eficacia de la estrategia territorial se expresa el nivel de satisfacción de la comunidad respecto del sistema preventivo. A este respecto, sobre el $60 \%$ de las personas le otorga notas sobre 6 a las alarmas en su vecindario, una medida del todo positiva. Es más, son muy escasas las notas bajo cuatro y en general la mayoría está por sobre la nota cinco. Como corolario, un $32 \%$ de los residentes encuestados calificó con nota siete a este sistema.

Gráfico 15: Satisfacción con las alarmas vecinales (Nota)

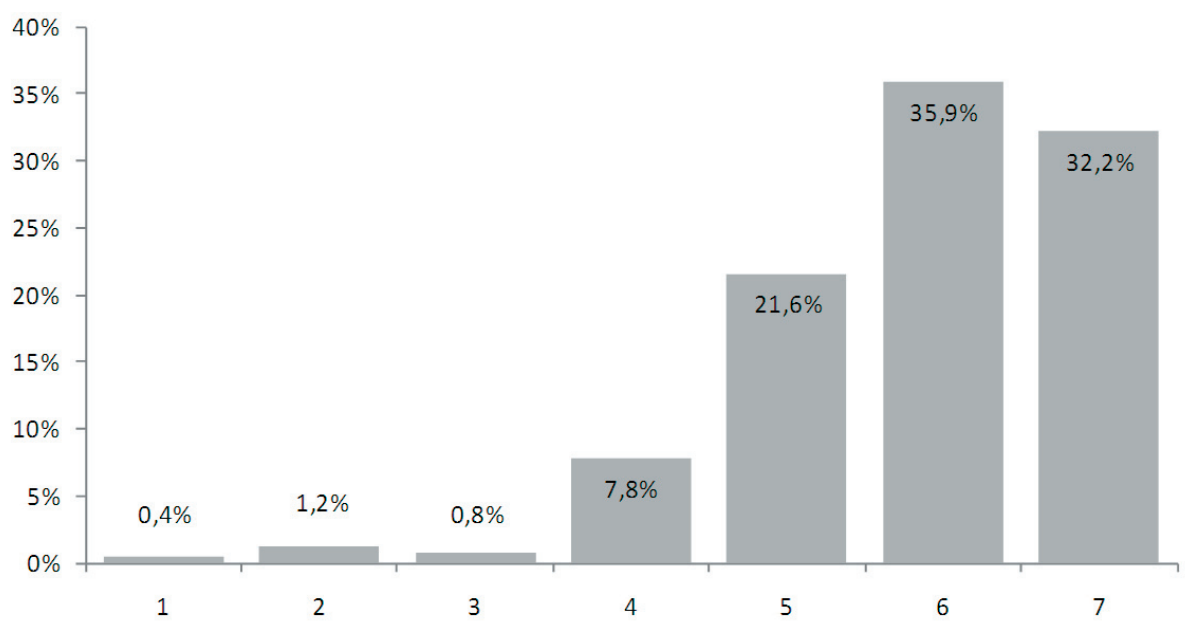

Fuente: autores a base de Encuesta de Victimización 2010 


\section{CONCLUSIONES}

Lo que podemos constatar después de analizar la opinión de los usuarios del sistema de alarmas residenciales en estos sectores de la comuna de Estación Central es la concreción de ciertos cambios, muy relacionados con los problemas que le dieron origen, es decir, en victimización e inseguridad. En efecto, habría una inicial pero clara obtención de resultados de la estrategia vecinal, junto con la aprobación de sus beneficiarios y el funcionamiento adecuado de la gestión comunitaria que lo soporta logrando naturalizar ciertas conductas en las personas; no obstante, observamos algunos problemas necesarios de abordar tanto para futuros proyectos de este tipo como para la misma intervención en esos sectores.

El hallazgo más relevante es que en ambos sectores analizados, correspondientes a tres villas altamente pobladas y con problemas de delincuencia (España, Fernando Gualda y Gustavo Guevara) se registraron disminuciones tanto en la victimización a hogares como en la percepción de inseguridad del barrio. Sobre todo en esta última medida, la que emerge como uno de los logros más claros conseguidos por la intervención.

Además existen valoraciones positivas respecto de la seguridad del barrio, respecto del conocimiento de iniciativas municipales de seguridad y una buena aprobación de las mismas.Dicho de otro modo, las políticas de seguridad municipales si importan y son conocidas y apreciadas por los vecinos.

Sin embargo, en una lectura más fina y focalizada, constatamos diferencias al interior de las mismas villas. En efecto, el primer sector observado -villa España- presenta resultados menos evidentes respeto del sistema preventivo, una disminución más leve de los indicadores de seguridad y menor aprobación de la gestión comunitaria del sistema, en comparación con los altos logros del segundo sector -villas Fernando Gualda y Gustavo Guevara- donde los apoyos, satisfacción y resultados son más apreciables.

Cabe destacar, que en el tema global de la seguridad en el espacio local de villa España la victimización se mantuvo entre la medición inicial y la evaluación posterior, no pudiéndole adjudicar una mejoría al sistema preventivo instalado. Del mismo modo, la percepción de inseguridad disminuyó, aunque levemente, en mismo barrio. El tipo de delito más frecuente sigue siendo el robo en hogares, tal como en la medición de base, pero su proporción disminuyó. Las personas de este sector calificaron positivamente la seguridad de su barrio (las notas 5 y 6 son las que predominan). Finalmente, los residentes tienen un alto nivel de conocimiento de las políticas de seguridad ejecutadas por su municipio y las aprueban y respaldan adecuadamente.

En el mismo tema global de la seguridad en el espacio local, el segundo sector de villas Fernando Gualda y Gustavo Guevara registró una disminución evidente de la victimización entre la medición inicial y la evaluación posterior. La percepción de inseguridad disminuyó considerablemente y tal como en el caso de villa España el 
tipo de delito más frecuente sigue siendo el robo en hogares, pero su proporción disminuyó. Igualmente, las personas calificaron positivamente la seguridad de su barrio (con notas mayoritarias de 5 y 6). Conjuntamente tienen un alto grado de conocimiento de las políticas de seguridad municipales $y$, tal como el primer sector evaluado, las aprueban altamente.

Una posible explicación a estas diferencias puede recaer en el entramado comunitario y la cohesión social existente en ambos sectores que diferenciamos territorial y analíticamente. En villa España, el sector con resultados moderados, la directiva de la junta de vecinos ha tenido dificultades en su validación ante la comunidad que representa, ni es sentida como referente por todos los habitantes de la villa. Desde nuestras aproximaciones cualitativas constatamos que no existen redes sólidas entre los vecinos de ese sector, a diferencia de las villas Fernando Gualda y Gustavo Guevara. También podemos encontrar explicaciones en la alta extensión geográfica del sector, lo que conspira contra un mayor compromiso de los vecinos pues existen lugares apartados que los mismos vecinos no sienten como propios, a diferencia del otro sector analizado. En la práctica la información sobre la gestión comunitaria del proceso no circula adecuadamente lo que dificulta la gestión de la estrategia.

Ahora bien, en cuanto al tema específico del sistema de alarmas comunitarias en ambos sectores analizados concluimos un nivel adecuado y alto de satisfacción, apoyo y valoración. De hecho, los habitantes evaluaron como una buena iniciativa esta estrategia de alarmas una vez transcurrido más de un año de ejecución. Las personas señalaron que es una ayuda evidente para prevenir la delincuencia, asignándole responsabilidad directa a esta estrategia.

Desde los beneficiarios pudimos conocer que no obstante la atribución de resultados y de apoyo, es un sistema preventivo de escaso uso por parte de los habitantes, lo que no invalida su instalación por cuanto son dispositivos de disuasión y planificados para la co-gestión, pensados en una utilización limitada y sólo en caso de necesidad.

Mismos agentes locales nos permiten concluir que a más de un año de ejecutada esta acción se redujo el problema que le dio origen, además que la percepción externa sobre el barrio es mejor que al inicio de la intervención y señalan que personas de otras villas de la comuna observan el sector en que viven como más seguro que antes.

En materia de exploración de dificultades, las personas señalan que mayoritariamente esto recae en la falta de organización de los vecinos y en la descoordinación con carabineros. Dos puntos sensibles de la estrategia y que coincide con los planteamientos de la literatura sobre prevención local, ya que en el centro de las acciones debe estar la articulación y la gestión colectiva no sólo de soluciones o respuestas a los problemas, sino de las prácticas sociales diarias, del seguimiento de las acciones colectivas y de su continuidad. La experiencia comparada muestra que el involucramiento en la esfera de la prevención incorpora 
los intereses de distintas subculturas organizacionales, en este caso de vecinos organizados y de policías, que compiten entre sí para orientar a su modo las políticas públicas locales, lo cual no sólo es legítimo y real sino que incluso conveniente si tienen como meta el logro de resultados en seguridad.

Comprobamos que se lograron resultados adicionales al objetivo de la política, perfectamente atribuibles a la intervención, puesto que la comunicación entre los vecinos mejoró sustancialmente, en un proxy de medición de eficacia de la política local; ya que el modelo de gestión comunitaria permite alcanzar esos fines. Igualmente existió un adecuado y muy adecuado uso de las alarmas por parte de los residentes, no registrándose alteraciones relevantes de su uso como podría suponerse con la implantación de dispositivos y elementos físicos que pueden permear la tranquilidad emocional de los sujetos. Kessler (2009) ha mostrado que una de las dificultades de estos dispositivos de prevención situacional tan próximos a las personas es que generan agobio y temor, caso contrario, en esta práctica social las personas logran tomar distancia y sentir mayores niveles de control de su seguridad, siendo de provecho para ellos. Finalmente existe un elevado grado de satisfacción con el sistema lo que augura sostenibilidad de la estrategia, un logro para cualquier política local en pleno desarrollo y anclada en la comunidad que vive y sufre problemas propios de inseguridad.

De modo general podemos señalar lo recomendado por Olavarría quien indica que "una de las características centrales de las estrategias preventivas consiste en que no sólo generan consecuencias sobre la violencia y el delito, sino que adicionalmente pueden fortalecer la democracia, la solidaridad y las redes sociales" (2007: 59). Esta estrategia localizada nos permite corroborar este supuesto.

\section{BIBLIOGRAFÍA}

Araya, J. (2009). Índice de vulnerabilidad social delictual. División de seguridad Pública, Gobierno de Chile.

Crawford, A. (1997). The Local Governance of Crime: Appeals to Community and Partnerships. Oxford: ClarendonPress.

Dammert, L. (2007). “Seguridad pública en América Latina: ¿Qué pueden hacer los gobiernos locales?”. En Revista Nueva Sociedad N² 212.

Edwards, A. y Gordon, H. (2009). "Comparando el gobierno de la seguridad en Europa”. En Urvio, Revista Latinoamericana de Seguridad Ciudadana. No. 6, Quito: FLACSO Sede Ecuador.

Instituto Chileno de Estudios Municipales. (2010). “Encuesta Nacional de Opinión Pública Municipal”. Universidad Autónoma de Chile. Octubre.

Kessler, G. (2009). El sentimiento de inseguridad. Sociología del temor al delito. Buenos Aires: Siglo XXI Editores. 
Manzano, L. (2009). Violencia en barrios críticos. Santiago: RIL Editores.

Ministerio del Interior (20I0). Política de Seguridad Pública, Chile Seguro. División de Seguridad Pública, Gobierno de Chile.

Muñoz, F. y Godoy, F. (2010). El rol del dirigente vecinal en los procesos de participación ciudadana institucionalizada: El caso de Estación Central”. En Revista Iberoamericana de Estudios Municipales. № 2.Universidad Autónoma de Chile.

Olavarría. M. (2007). "Delito y Violencia. Causas. Consecuencias y Estrategias de Prevención”. En Documento de Apoyo Docente N²0, INAP Universidad de Chile.

Rodríguez, J. Gil,J.y García, E. ( 1999). Metodología de la investigación cualitativa. Málaga: Aljibe.

Vanderschueren, F. (2007). Modelo Democráticos de Prevención del Delito. Santiago: U. Alberto Hurtado. 